\title{
Loneliness and Risk for Cardiovascular Disease: Mechanisms and Future Directions
}

\author{
Elise Paul ${ }^{1}$ (D) Feifei Bu ${ }^{1} \cdot$ Daisy Fancourt ${ }^{1}$
}

Accepted: 9 March 2021 / Published online: 7 May 2021

(C) The Author(s) 2021

\begin{abstract}
Purpose of review In this review, we synthesise recent research on the association between loneliness and cardiovascular disease (CVD). We present evidence for mechanisms underlying this association and propose directions for future research.

Recent findings Loneliness is related to increased risk of early mortality and CVD comparable to other well-established risk factors such as obesity or smoking.

Summary Loneliness has been linked to higher rates of incident CVD, poorer CVD patient outcomes, and early mortality from CVD. Loneliness likely affects risk for these outcomes via health-related behaviours (e.g. physical inactivity and smoking), biological mechanisms (e.g. inflammation, stress reactivity), and psychological factors (e.g. depression) to indirectly damage health.
\end{abstract}

Keywords Loneliness $\cdot$ Cardiovascular disease $\cdot$ Epidemiology $\cdot$ Health policy $\cdot$ Health promotion

\section{Introduction}

Accumulating evidence suggests that psychosocial risk factors such as loneliness are significant contributors to a wide range of negative health outcomes, including early all-cause mortality $[1,2]$, incident cardiovascular disease (CVD) [3•], and allcause mortality in patients with CVD [4]. There are several indications that loneliness, or the subjective distress resulting from a discrepancy between desired and perceived social relationships [5], is widespread. National surveys in the UK find that $5-7 \%$ of adults feel lonely often or always [6-8]. Others find that nearly half (43\%) of older adults (aged 60 and over)

This article is part of the Topical Collection on Psychological Aspects of Cardiovascular Diseases

Daisy Fancourt

d.fancourt@ucl.ac.uk

Elise Paul

e.paul@ucl.ac.uk

Feifei Bu

f.bu@ucl.ac.uk

1 Department of Behavioural Science and Health, University College London, 1-19 Torrington Place, London WC1E 7HB, UK report feeling lonely [9]. Many of the groups more likely to be lonely are also those at an increased risk for CVD. For example, loneliness is more common amongst individuals of lower socioeconomic status (SES), poorer health, older age, and cognitive and physical impairment $[10,11]$. These factors are in turn associated with higher CVD risk [12-16]. This has led to loneliness itself being identified as a public health concern [17] and developments in policy such as the establishment of a Minister for Loneliness in the UK in 2018, and the national roll-out of "social prescribing" in the NHS, the goal of which is for every general practitioner (GP) to be able to refer patients experiencing loneliness to voluntary services and community activities by the year 2023 [18].

A related but more objective construct, social isolation, has been operationalised as the number of persons with whom an individual has contact or lives, engagement in volunteering or work, and membership in social organisations or clubs [19, 20]. Although social isolation and loneliness are related and can occur together, they can also be experienced independently of one another [21]. Meta-analyses find that social isolation has comparable associations with all-cause mortality $[1 \bullet]$ and CVD to loneliness [3•]. Whilst this review focuses specifically on loneliness rather than social isolation in relation to CVD, where studies have accounted for both, we report both sets of findings for comparison. 
In this review, we provide a broad overview of current knowledge concerning whether and how loneliness influences risk for CVD incidence, prognosis, and CVD-related mortality. We begin by focusing on evidence directly linking loneliness with these outcomes. We then consider (1) psychological, (2) biological, and (3) behavioural mechanisms by which loneliness may increase the risk for CVD, emphasising findings from methodologically stronger studies when available. We conclude with a summary of the limitations of existing research as well as recommendations for future work in this area.

\section{Loneliness and cardiovascular disease: the evidence}

Evidence linking loneliness with CVD comes from prospective studies (and meta-analyses of these studies) using population-based and CVD patient samples. In the most recent large-scale meta-analysis linking these outcomes, the pooled relative risk for incident coronary heart disease (CHD) and stroke in individuals with high versus low levels of loneliness or social isolation was 1.29 (95\% confidence interval $(\mathrm{CI})=1.04$ to 1.59 ) in 16 longitudinal studies [3•]. There were no significant differences in the magnitude of risk when studies examining either social isolation or loneliness were pooled separately, or in relative risk for CVD outcomes by gender, risk of confounding, or risk of measurement error.

Since this meta-analysis, other studies have repeated these findings. For example, using data from adults aged 40-69 in the large prospective population-based UK Biobank study, Hakulinen et al [22] found that in separate regression models, both loneliness and social isolation were associated with increased risk for incident acute myocardial infarction and first stroke when adjusting for demographics. Similarly, a large prospective Danish study showed independent associations of both loneliness (hazard ratio $(\mathrm{HR})=1.20 ; 95 \% \mathrm{CI}=1.03$ to 1.40$)$ and social isolation $(\mathrm{HR}=1.23 ; 95 \% \mathrm{CI}=1.04$ to 1.46) with CVD [23].

However, there have been mixed findings as to whether subjective feelings of loneliness or the objective construct of social isolation is more important for CVD. A 10-year followup study of Taiwanese adults with CVD found evidence for the role of social isolation $(\mathrm{HR}=1.16 ; 95 \% \mathrm{CI}=1.07$ to 1.27 ) but not loneliness ( $\mathrm{HR}=0.92 ; 95 \% \mathrm{CI}=0.80$ to 1.06$)$ with increased risk of all-cause mortality after accounting for both simultaneously and adjusting for other established risk factors [4]. Similar findings were reported using data from the Danish DenHeart study of 13,000 CVD patients, where living alone but not loneliness was associated with risk for cardiac events (myocardial infarction, stroke, ventricular tachycardia/ ventricular fibrillation, and cardiac arrest) assessed from national registers in men $(\mathrm{HR}=1.39 ; 95 \% \mathrm{CI}=1.05$ to 1.85$)$ at 1-year follow-up [24]. Furthermore, two other sets of analyses using data from the large population-based English Longitudinal Study of Ageing (ELSA) found that loneliness, but not indices of social isolation, was associated with increased risk for incident CVD outcomes including mortality. In the first, loneliness was associated with $30 \%$ higher risk of CVD incidence at 5-year follow-up, whilst social isolation was not [25]. A second study using the ELSA data found that loneliness but not social isolation associated with self-reported and hospital admission CVD events using objective registry data independent of sociodemographic factors [19]. So, studies remain inconclusive as to whether loneliness or social isolation are stronger predictors of CVD and CVD-related outcomes.

It is also possible that not everyone is at equal risk from social factors. For example, there have been some differential findings for men and women with respect to loneliness. Using data from the Swedish Gothenburg H70 Birth Cohort Studies, loneliness was a predictor of cardiovascular mortality in women $(\mathrm{HR}=2.42 ; 95 \% \mathrm{CI}=1.04-5.65)$, but not in men ( $\mathrm{HR}=$ 1.52 ; 95\% CI $=0.78-2.96$ ) independent of living alone [26]. Additionally, in a prospective study of population-based Finnish men (ages 42-61) who were followed for an average of 23 years, loneliness but not social isolation predicted CVD mortality $(\mathrm{HR}=1.12 ; 95 \% \mathrm{CI}=1.01$ to 1.24$)$, even whilst adjusting for depressive symptoms and other confounding variables [27].

In sum, the majority of studies reviewed support subjective feelings of loneliness independent of the objective measure of social isolation as an important risk factor for incident CVD and CVD events in CVD patients. Whilst the specifics of the evidence are somewhat mixed, variation across study outcomes may depend in part on the covariates included in analyses. But there is nonetheless repeated evidence from both self-reported and objective registry data of a relationship between social factors and various CVD outcomes.

\section{Mechanisms linking loneliness and CVD}

To date, a number of psychological, biological, and behavioural pathways to health and mortality from loneliness have been identified $[28,29]$. Several researchers who have conceptualised the relationships amongst these variables begin with the observation that humans are social creatures, and that proximity to other humans is necessary for survival $[30,31]$. Perceived social isolation is therefore a source of threat and danger, which activates behavioural and psychological mechanisms via biological and molecular pathways [29-31]. These mechanisms in turn lead to increased risk for premature mortality and disease [28, 32]. Identifying these pathways is crucial for developing appropriate interventions and prevention targets [28•]. Below, we review the 
recent evidence for these pathways whilst acknowledging that the mechanisms also likely interact with each other and operate bidirectionally.

\section{Psychological mechanisms}

Loneliness is an established risk factor for mental ill health in meta-analyses, including depressive [33] and anxious symptoms [34], and thoughts of suicide and suicidal behaviour [11, $34,35]$.

Depression in particular is hypothesised to be a mechanism linking loneliness with poor health outcomes such as CVD $[28,34]$. Displays of depressive behaviour may provide a non-verbal signal to others that support and connection are needed [30]. Whilst depression and loneliness have been hypothesised to be bidirectional, recent research has specifically identified loneliness as a precursor to major depressive disorder (MDD) 2 years later, but has not found the association operating the other way [36]. However, it is likely that depression is not the sole mediator between loneliness and CVD as two recent prospective large studies found that the relationship between loneliness and CVD mortality persisted even whilst adjusting for depressive symptoms [19, 27].

Another psychological mechanism that could explain the link between loneliness and CVD is stress. Stress is a key aspect of the experience and definition of loneliness [5]. Humans are inherently social animals and perceived isolation may activate a stress response designed to facilitate survival in the short-term by seeking proximity to other humans, but also by producing hypervigilance to threats to compensate for the lack of mutual protection [30,31]. Psychological stress has been shown to result from loneliness, along with biological stress responses (see "biological mechanisms" below), and stress-related health behaviours (see "behavioural mechanisms") [37, 38]. Increased stress can in turn lead to compromised physiological resilience [39]. Excessive worries over time that are not attenuated by the removal of stressors can also become more generalised anxiety, which has been shown to be bidirectionally related to loneliness [36].

Additionally, research has also examined other negative behaviours including anger and hostility in relation to loneliness, finding that these behaviours are heightened in lonely individuals, perhaps in part because increased vigilance for social threats in the absence of perceived social connectedness may protect lonely individuals in the short term [30]. However, these negative states, along with stress and depression, can also act as a short-term precipitant of major cardiac events such as acute coronary syndrome [40, 41]. Therefore, evidence suggests that negative moods, stress, and poor mental health are all pathways connecting loneliness to cardiovascular events.

\section{Biological mechanisms: neuroendocrine, immune, cardiometabolic, physiological, and epigenetic}

Evidence for biological pathways through which loneliness impacts CVD comes from both human and animal studies $[30,32]$. Mortality risk from loneliness remains elevated even when controlling for psychological and behavioural factors, suggesting a role for biological mechanisms [28•]. Underlying many of these biological pathways are psychological mechanisms discussed above including depression and chronic elevated stress [28, 29], This is likely because hormonal, vascular, and immune responses are set off by the danger to one's survival from perceived social isolation and loneliness $[28,32$, $40,42]$. These biological responses in turn can, when chronic and sustained, lead to adverse health consequences which place the individual at risk for premature mortality and disease development. Some of these key biological pathways are outlined below.

Neuroendocrine pathways The stress associated with loneliness has led to much research focused on atypical (exaggerated or blunted) neuroendocrine responses to stress as a major pathway through which subjective feelings of loneliness influence CVD risk [28, 32, 42, 43]. Loneliness activates the body's central stress response system - the hypothalamic pituitary adrenal (HPA) axis - in order to prepare the body to deal with potential threats that may occur as a result of perceived social isolation $[32,42]$. The HPA axis sets into motion a "flight or fight" response by secreting the stress hormone cortisol, which typically shows higher levels upon morning awakening and a blunted circadian rhythm across the day [42•]. Over time, chronic stress can lead to chronically elevated cortisol levels, which are associated with CVD incidence and poorer prognosis, as well as CVD risk factors such as diabetes and systolic blood pressure [44].

The majority of the available evidence supports an exaggerated HPA response in association with loneliness; larger increases in cortisol upon awakening, higher concentrations of circulating cortisol, and diminished sensitivity of glucocorticoid receptors have all been found in lonely individuals [32•]. Specifically in reaction to stressful events, momentary, day-to-day sampling studies have found higher levels of cortisol reactivity in lonely compared to nonlonely individuals $[45,46]$. However, there is still some inconsistency in the literature: a systematic review of studies measuring neuroendocrine responses to acute laboratory-induced stressors such as giving a public speech found that although most studies reviewed reported exaggerated stress responses in lonelier individuals, one study found blunted cortisol production in lonelier women but not men, whilst two others reported no difference in cortisol levels between lonely and non-lonely individuals [43]. 
Responses have also not been as clearly found for other hormones. Studies examining loneliness and neuropeptides have found no association with catecholamines (e.g. epinephrine, norepinephrine, dopamine) secreted in response to signals from the sympathetic nervous system during acutely stressful events $[47,48]$. Therefore, further research on the role of stress hormones in mediating the relationship between loneliness and CVD using larger longitudinal studies is needed.

Immune function A second possible biological mechanism linking loneliness with CVD is weakened immunity through increased inflammation $[28,31,32]$. Cytokines (inflammatory proteins within the immune system) not only coordinate the body's inflammatory response, but also initiate so-called "sickness behaviours", which include fatigue and social withdrawal and are a part of individuals' response to illness [31, 49]. The resulting inflammation may enable sick individuals to prepare for the possibility of infection or harm $[31,50]$. Therefore, proinflammatory response that occurs in loneliness may be seen as a protection in the short term against threats to safety, as humans who are disconnected from others are more vulnerable to illness [31]. However, this inflammation may also increase CVD risk when sustained.

In terms of specific cytokines and other inflammatory markers associated with loneliness, a systematic review of the evidence linking loneliness and inflammatory reactions to acute laboratory-induced stress (e.g. a performance task or pharmacological induction with amphetamines) found loneliness to be associated with increased proinflammatory cytokines and glycoproteins such as IL-6, tumour necrosis factor alpha (TNFa), interleukin-1 beta (IL-1B), monocyte chemoattractant protein 1 (MCP-1), and fibrinogen [43]. However, a more recent meta-analysis focusing on observational studies of loneliness, social isolation, and inflammation found higher levels of interleukin-6 (IL-6) but not fibrinogen in association with loneliness [51]. This study also found no relationship with the inflammatory marker C-reactive protein (CRP) [51]. Even though CRP is a biomarker associated with depression (which we have already discussed as one psychological pathway linking loneliness with CVD) [52], the relationship between CRP and loneliness has also not been shown by other studies, including a study that found no evidence that CRP acts as a mediator between loneliness and depression [53], and another study finding that loneliness was associated with higher inflammatory markers such as insulin-like growth factor 1 (IGF-1) but not CRP or fibrinogen, even though social isolation was related to these factors [54]. So, it appears that only some inflammatory markers are related to loneliness.

Additionally, in their recent review, Li and Xia [32] argued that another biological mechanism underlying the association between psychosocial stress due to loneliness and CVD is oxidative stress. Longitudinal research on this topic is needed given evidence for a bi-directional relationship between loneliness and immune response [55]. However, it highlights the breadth of immune markers now being considered in relation to loneliness.

Cardiometabolic response A third possible pathway through which loneliness increases the risk for CVD is cardiometabolic changes such as elevated blood pressure $[28,43]$ and heart rate variability [43]. In a recent meta-analysis, loneliness was related to elevated diastolic blood pressure, increased vascular resistance, and lower heart rate variability in response to acute laboratory-based stressors [43]. A more recent narrative review reported lonely individuals to have greater total peripheral vascular resistance and increased risk of hypertension [32]. However, findings from the ELSA epidemiological study (not included in the Brown et al. [43] review) showed that social isolation but not loneliness was associated with increases in both systolic and diastolic blood pressure [20]. Since these reviews, one study of healthy young women (18-29 years) found that those with greater levels of chronic loneliness had lower resting HRV and significantly greater increases in HRV after exposure to laboratory-induced state of loneliness [56]. Women who were chronically lonely also showed blunted HRV reactivity to a cognitive challenge task [56].

Psychosocial stress associated with loneliness may also influence CVD via changes in gut microbiota. Activation of the HPA axis initiates an immune response that in turn decreases microbial diversity, and leads to further HPA axis activation [57]. These stress-induced changes in gut microbiota have been shown to increase risk for CVD in clinical associations in human studies and experimental studies in animals $[58,59]$. However, research has yet to determine whether the stress associated with loneliness in particular induces changes in gut microbiota which are in turn associated with CVD.

In considering other biological pathways, small genetic contributions to loneliness have been reported [60] and chronic social isolation in mice and rats has been shown to lead to epigenetic changes in the brain [61,62]. However, research on whether loneliness induces epigenetic changes in humans is needed, particularly changes in the epigenome that are associated with CVD risk.

\section{Behavioural mechanisms}

Finally, the quality of one's social relationships may have an indirect effect on CVD by promoting healthy behaviours such as exercise, healthy eating, and not smoking or abusing alcohol [63]. Feeling lonely on the other hand may impair the capacity to self-regulate and avoid negative behaviours such as excessive alcohol use which reduce stress and tension in the 
short-term, but are ultimately harmful for health in the longterm [30]. Evidence supporting these behavioural mechanisms comes from studies showing loneliness is associated with a lack of physical activity [20,64], smoking [20, 65, 66], poor diet [65], and problematic alcohol use [67-69].

In other studies, the association between loneliness and CVD has been found to be attenuated when adjusting for behavioural factors that could lie on the causal pathway and thereby act as pathways to CVD. In models that adjusted for potential mechanisms such as physical inactivity, smoking, and body mass index, the relationship between loneliness and mortality has been shown to be attenuated in participants who had earlier acute myocardial infarction [22]. However, one recent longitudinal study of older adults (ELSA) showed that the impact of loneliness on CVD persisted, even when accounting for physical inactivity, smoking, and alcohol use [19], suggesting that these behavioural factors may only partially explain the relationship with CVD-related outcomes and also that more research is needed to establish the direction of these associations. Further, whether it is loneliness or social isolation that has the strongest association with health behaviours is still being questioned as another study using data from the ELSA cohort found that social isolation but not loneliness at baseline was associated with a number of health risk behaviours (e.g. decreased likelihood of regular physical activity, lower fruit and vegetable intake, smoking) [70]. Lonely individuals who smoked were, however, less likely to have stopped smoking at follow-up than non-lonely individuals [70].

In sum, alongside psychological and biological pathways connecting loneliness to CVD risks, there are also a number of potential behavioural pathways that likely interact with other pathways. For example, sedentary behaviours are themselves associated with higher depression and inflammatory response $[71,72]$. This highlights both the range of mechanisms connecting loneliness to CVD but also the interconnectedness of these mechanisms.

\section{Conclusions and implications}

Research demonstrates that loneliness is an independent and modifiable risk factor for CVD. The available evidence implicates psychological factors such as stress, depression, atypical physiological reactivity, and neuroendocrine responses to stress, increased inflammation, and harmful behaviours such as smoking, drinking alcohol, and poor diet as possible mechanisms.

Evidence of effective interventions for loneliness is limited [9]. A review of interventions to reduce social isolation and loneliness in older adults found that whilst most did report effectiveness, the quality of evidence was weak [73]. Although participants in social-prescribing interventions (e.g. group exercise, arts classes) report satisfaction with the programmes and reduced feelings of loneliness $[74,75]$, there have to date not been studies examining mortality or CVD outcomes of these non-medical interventions (social prescribing) [28•]. Therefore, in addition to deeper exploration of the magnitude of the relationship between loneliness and CVD risk amongst different populations (including groups at low and high risk of developing CVD) and the mechanisms underlying this relationship (in particular how combinations of mechanisms can act synergistically with one another), future research is also encouraged to investigate interventions that could help to reduce the negative health effects of loneliness. Such research could play an important role in the design of holistic care strategies for people at risk of or living with CVD and help to reduce the burden of managing CVD for health and social care services.

Funding This paper was supported by the MARCH Mental Health Network funded by the Cross-Disciplinary Mental Health Network Plus initiative supported by UK Research and Innovation [ES/S002588/1]. Additionally, DF is supported by the Wellcome Trust [205407/Z/16/Z] and the project received additional funding support through the ESRC project WELLCOMM [ES/T006994/1] and the Leverhulme Trust [PLP2018-007].

\section{Declarations}

Conflict of Interest The authors have no conflicts of interest to declare.

Human and Animal Rights and Informed Consent This article does not contain any studies with human or animal subjects performed by any of the authors.

Open Access This article is licensed under a Creative Commons Attribution 4.0 International License, which permits use, sharing, adaptation, distribution and reproduction in any medium or format, as long as you give appropriate credit to the original author(s) and the source, provide a link to the Creative Commons licence, and indicate if changes were made. The images or other third party material in this article are included in the article's Creative Commons licence, unless indicated otherwise in a credit line to the material. If material is not included in the article's Creative Commons licence and your intended use is not permitted by statutory regulation or exceeds the permitted use, you will need to obtain permission directly from the copyright holder. To view a copy of this licence, visit http://creativecommons.org/licenses/by/4.0/.

\section{References}

Papers of particular interest, published recently, have been highlighted as:

- Of importance

1. Holt-Lunstad J, Smith TB, Baker M, Harris T, Stephenson D. Loneliness and social isolation as risk factors for mortality: a meta-analytic review. Perspect Psychol Sci. 2015;10:227-37 
Important because it is the most recent meta-analysis on loneliness and mortality.

2. Rico-Uribe LA, Caballero FF, Martín-María N, Cabello M, AyusoMateos JL, Miret M. Association of loneliness with all-cause mortality: a meta-analysis. PLoS One. 2018;13:e0190033.

3. Valtorta NK, Kanaan M, Gilbody S, Ronzi S, Hanratty B. Loneliness and social isolation as risk factors for coronary heart disease and stroke: systematic review and meta-analysis of longitudinal observational studies. Heart. 2016;102:1009-16 Important because it is the most recent meta-analysis on loneliness and CVD.

4. Yu B, Steptoe A, Chen L-J, Chen Y-H, Lin C-H, Ku P-W. Social isolation, loneliness, and all-cause mortality in patients with cardiovascular disease: a 10-year follow-up study. Psychosom Med. 2020;82:208-14.

5. Peplau LA, Perlman D. Perspectives on loneliness. In: Loneliness: a sourcebook of current theory, research and practice. New York: John Wiley; 1982. p. 1-17.

6. Li LZ, Wang S. Prevalence and predictors of general psychiatric disorders and loneliness during COVID-19 in the United Kingdom. Psychiatry Res. 2020;291:113267.

7. Office for National Statistics (2018) Loneliness-what characteristics and circumstances are associated with feeling lonely?

8. Office for National Statistics. Coronavirus and loneliness. Great: Britain - Office for National Statistics; 2020.

9. National Academies of Sciences, Engineering, and Medicine (2020) Social isolation and loneliness in older adults: opportunities for the health care system. https://doi.org/10.17226/25663

10. Hajek A, König H-H. Which factors contribute to loneliness among older Europeans? Findings from the survey of health, ageing and retirement in Europe: determinants of loneliness. Arch Gerontol Geriatr. 2020;89:104080.

11. Solmi M, Veronese N, Galvano D, Favaro A, Ostinelli EG, Noventa V, et al. Factors associated with loneliness: an umbrella review of observational studies. J Affect Disord. 2020;271:131-8.

12. Calderón-Larrañaga A, Vetrano DL, Ferrucci L, Mercer SW, Marengoni A, Onder G, et al. Multimorbidity and functional impairment-bidirectional interplay, synergistic effects and common pathways. J Intern Med. 2019;285:255-71.

13. de Mestral C, Stringhini S. Socioeconomic status and cardiovascular disease: an update. Curr Cardiol Rep. 2017;19:115.

14. Ferrucci L, Fabbri E. Inflammageing: Chronic inflammation in ageing, cardiovascular disease, and frailty. Nat Rev Cardiol. 2018;15: 505-22.

15. Lahey R, Khan SS. Trends in obesity and risk of cardiovascular disease. Curr Epidemiol Rep. 2018;5:243-51.

16. Rosengren A, Smyth A, Rangarajan S, Ramasundarahettige C, Bangdiwala SI, AlHabib KF, et al. Socioeconomic status and risk of cardiovascular disease in 20 low-income, middle-income, and high-income countries: the Prospective Urban Rural Epidemiologic (PURE) study. Lancet Glob Health. 2019;7:e74860.

17. Jeste DV, Lee EE, Cacioppo S. Battling the modern behavioral epidemic of loneliness: suggestions for research and interventions. JAMA Psychiatry. 2020;77:553-4.

18. HM Government (2018) PM launches Government's first loneliness strategy. In: GOV.UK. https:/www.gov.uk/government/news/ pm-launches-governments-first-loneliness-strategy. Accessed 29 Oct 2020

19. $\mathrm{Bu} \mathrm{F}$, Zaninotto $\mathrm{P}$, Fancourt D. Longitudinal associations between loneliness, social isolation and cardiovascular events. Heart. 2020;106:1394-9.

20. Shankar A, McMunn A, Banks J, Steptoe A. Loneliness, social isolation, and behavioral and biological health indicators in older adults. Health Psychol: official journal of the Division of Health
Psychology, American Psychological Association. 2011;30:37785.

21. Perissinotto CM, Covinsky KE (2014) Living alone, socially isolated or lonely - what are we measuring? Springer

22. Hakulinen C, Pulkki-Raback L, Virtanen M, Jokela M, Kivimäki $\mathrm{M}$, Elovainio M. Social isolation and loneliness as risk factors for myocardial infarction, stroke and mortality: UK Biobank cohort study of 479054 men and women. Heart. 2018;104:1536-42.

23. Christiansen J, Lund R, Qualter P, Andersen CM, Pedersen SS, Lasgaard M. Loneliness, social isolation, and chronic disease outcomes. Ann Behav Med. 2020. https://doi.org/10.1093/abm/ kaaa044.

24. Christensen AV, Juel K, Ekholm O, Thrysøe L, Thorup CB, Borregaard B, et al. Significantly increased risk of all-cause mortality among cardiac patients feeling lonely. Heart. 2020;106:1406.

25. Valtorta NK, Kanaan M, Gilbody S, Hanratty B. Loneliness, social isolation and risk of cardiovascular disease in the English Longitudinal Study of Ageing. Eur J Prev Cardiol. 2018;25:138796.

26. Novak M, Waern M, Johansson L, Zettergren A, Ryden L, Wetterberg $\mathrm{H}$, et al. Cardiovascular and all-cause mortality attributable to loneliness in older Swedish men and women. BMC Geriatr. 2020;20:201.

27. Kraav S-L, Awoyemi O, Junttila N, Vornanen R, Kauhanen J, Toikko T, Lehto SM, Hantunen S, Tolmunen T (2020) The effects of loneliness and social isolation on all-cause, injury, cancer, and CVD mortality in a cohort of middle-aged Finnish men. A prospective study. Aging Mental Health 1-10

28. Hodgson S, Watts I, Fraser S, Roderick P, Dambha-Miller H. Loneliness, social isolation, cardiovascular disease and mortality: a synthesis of the literature and conceptual framework. J R Soc Med. 2020;113:185-92 Key article because it provides a framework for mechanisms linking loneliness with cardiovascular disease.

29. Xia N, Li H. Loneliness, social isolation, and cardiovascular health Antioxid Redox Signal. 2018;28:837-51.

30. Cacioppo S, Capitanio JP, Cacioppo JT. Toward a neurology of loneliness. Psychol Bull. 2014;140:1464-504.

31. Eisenberger NI, Moieni M, Inagaki TK, Muscatell KA, Irwin MR. In sickness and in health: the co-regulation of inflammation and social behavior. Neuropsychopharmacology. 2017;42:242-53.

32. $\mathrm{Li} \mathrm{H}, \mathrm{Xia} \mathrm{N}$. The role of oxidative stress in cardiovascular disease caused by social isolation and loneliness. Redox Biol. 2020;37: 101585 Key article because it provides a framework for biological mechanisms linking loneliness with cardiovascular disease.

33. Lee SL, Pearce E, Ajnakina O, Johnson S, Lewis G, Mann F, Pitman A, Solmi F, Sommerlad A, Steptoe A (2020) The association between loneliness and depressive symptoms among adults aged 50 years and older: a 12-year population-based cohort study. Lancet Psychiatry

34. Park C, Majeed A, Gill H, Tamura J, Ho RC, Mansur RB, et al. The effect of loneliness on distinct health outcomes: a comprehensive review and meta-analysis. Psychiatry Res. 2020;294:113514.

35. McClelland H, Evans JJ, Nowland R, Ferguson E, O'Connor RC. Loneliness as a predictor of suicidal ideation and behaviour: a systematic review and meta-analysis of prospective studies. J Affect Disord. 2020;274:880-96.

36. Domènech-Abella J, Mundó J, Haro JM, Rubio-Valera M. Anxiety, depression, loneliness and social network in the elderly: longitudinal associations from The Irish Longitudinal Study on Ageing (TILDA). J Affect Disord. 2019;246:82-8.

37. Cacioppo JT, Hawkley LC, Crawford LE, Ernst JM, Burleson MH, Kowalewski RB, et al. Loneliness and health: potential mechanisms. Psychosom Med. 2002;64:407-17. 
38. Hawkley LC, Burleson MH, Berntson GG, Cacioppo JT. Loneliness in everyday life: cardiovascular activity, psychosocial context, and health behaviors. J Pers Soc Psychol. 2003;85:105-20.

39. Hawkley LC, Cacioppo JT. Aging and loneliness: Downhill quickly? Curr Dir Psychol Sci. 2007;16:187-91.

40. Kivimäki M, Steptoe A. Effects of stress on the development and progression of cardiovascular disease. Nat Rev Cardiol. 2018;15: 215-29.

41. Whooley MA, Wong JM. Depression and cardiovascular disorders. Ann Rev Clin Psychol. 2013;9:327-54.

42. Quadt L, Esposito G, Critchley HD, Garfinkel SN. Brain-body interactions underlying the association of loneliness with mental and physical health. Neurosci Biobehav Rev. 2020;116:283-300 Key article because it provides a framework for mechanisms linking loneliness with cardiovascular disease.

43. Brown EG, Gallagher S, Creaven AM. Loneliness and acute stress reactivity: a systematic review of psychophysiological studies. Psychophysiology. 2018;55:e13031.

44. Iob E, Steptoe A. Cardiovascular disease and hair cortisol: a novel biomarker of chronic stress. Curr Cardiol Rep. 2019;21:116.

45. Doane LD, Adam EK. Loneliness and cortisol: momentary, day-today, and trait associations. Psychoneuroendocrinology. 2010;35: 430-41.

46. Drake EC, Sladek MC, Doane LD. Daily cortisol activity, loneliness, and coping efficacy in late adolescence: a longitudinal study of the transition to college. Int J Behav Dev. 2016;40:334-45.

47. Edwards KM, Bosch JA, Engeland CG, Cacioppo JT, Marucha PT. Elevated macrophage migration inhibitory factor (MIF) is associated with depressive symptoms, blunted cortisol reactivity to acute stress, and lowered morning cortisol. Brain Behav Immun. 2010;24:1202-8.

48. Gevonden M, Booij J, van den Brink W, Heijtel D, van Os J, Selten JP. Increased release of dopamine in the striata of young adults with hearing impairment and its relevance for the social defeat hypothesis of schizophrenia. JAMA Psychiatry. 2014;71:1364-72.

49. Dantzer R, Kelley KW. Twenty years of research on cytokineinduced sickness behavior. Brain Behav Immun. 2007;21:153-60.

50. Leschak CJ, Eisenberger NI. Two distinct immune pathways linking social relationships with health: inflammatory and antiviral processes. Psychosom Med. 2019;81:711-9.

51. Smith K, Gavey S, Ridell NE, Kontari P, Victor C. The association between loneliness, social isolation and inflammation: a systematic review and meta-analysis. Neurosci Biobehav Rev. 2020;112:519-41.

52. Osimo EF, Baxter LJ, Lewis G, Jones PB, Khandaker GM. Prevalence of low-grade inflammation in depression: a systematic review and meta-analysis of CRP levels. Psychol Med. 2019;49: 1958-70

53. Vingeliene S, Hiyoshi A, Lentjes M, Fall K, Montgomery S. Longitudinal analysis of loneliness and inflammation at older ages: English longitudinal study of ageing. Psychoneuroendocrinology. 2019;110:104421.

54. Walker E, Ploubidis G, Fancourt D. Social engagement and loneliness are differentially associated with neuro-immune markers in older age: time-varying associations from the English Longitudinal Study of Ageing. Brain Behav Immun. 2019;82: 224-9.

55. Cole SW, Capitanio JP, Chun K, Arevalo JM, Ma J, Cacioppo JT. Myeloid differentiation architecture of leukocyte transcriptome dynamics in perceived social isolation. Proc Natl Acad Sci. 2015;112: 15142-7.

56. Roddick CM, Chen FS. Effects of chronic and state loneliness on heart rate variability in women. Ann Behav Med. 2020. https://doi. org/10.1093/abm/kaaa065.
57. Makris AP, Karianaki M, Tsamis KI, Paschou SA. The role of the gut-brain axis in depression: endocrine, neural, and immune pathways. Hormones. 2020;20:1-12. https://doi.org/10.1007/s42000020-00236-4.

58. Witkowski M, Weeks TL, Hazen SL. Gut microbiota and cardiovascular disease. Circ Res. 2020;127:553-70.

59. Kazemian N, Mahmoudi M, Halperin F, Wu JC, Pakpour S. Gut microbiota and cardiovascular disease: opportunities and challenges. Microbiome. 2020;8:36.

60. Spithoven W, Cacioppo S, Goossens L, Cacioppo JT. Genetic contributions to loneliness and their relevance to the evolutionary theory of loneliness. Perspect Psychol Sci. 2019;14:3.

61. Bludau A, Royer M, Meister G, Neumann ID, Menon R. Epigenetic regulation of the social brain. Trends Neurosci. 2019;42:471-84.

62. Arzate-Mejia R, Lottenbach Z, Schindler V, Jawaid A, Mansuy I (2020) Long-term impact of social isolation and molecular underpinnings. Front Gen

63. Cacioppo JT, Patrick W (2008) Loneliness: human nature and the need for social connection. W W Norton \& Co

64. Schrempft S, Jackowska M, Hamer M, Steptoe A. Associations between social isolation, loneliness, and objective physical activity in older men and women. BMC Public Health. 2019;19:74.

65. Leigh-Hunt N, Bagguley D, Bash K, Turner V, Turnbull S, Valtorta $\mathrm{N}$, et al. An overview of systematic reviews on the public health consequences of social isolation and loneliness. Public Health. 2017;152:157-71.

66. Wootton RE, Greenstone HS, Abdellaoui A, Denys D, Verweij KJ, Munafò MR, Treur JL (2020) Bidirectional effects between loneliness, smoking and alcohol use: evidence from a Mendelian randomization study. Addiction

67. Canham SL, Mauro PM, Kaufmann CN, Sixsmith A. Association of alcohol use and loneliness frequency among middle-aged and older adult drinkers. J Aging Health. 2016;28:267-84.

68. Purser GL (2020) The combined effect of functional independence, loneliness, and social engagement on older adult drinking levels. J Soc Work Pract Addict 1-12

69. Segrin C, McNelis M, Pavlich CA. Indirect effects of loneliness on substance use through stress. Health Commun. 2018;33:513-8.

70. Kobayashi LC, Steptoe A. Social isolation, loneliness, and health behaviors at older ages: longitudinal cohort study. Ann Behav Med. 2018;52:582-93.

71. Hamer M, Poole L, Messerli-Bürgy N. Television viewing, Creactive protein, and depressive symptoms in older adults. Brain Behav Immun. 2013;33:29-32

72. Paolucci EM, Loukov D, Bowdish DM, Heisz JJ. Exercise reduces depression and inflammation but intensity matters. Biol Psychol. 2018;133:79-84.

73. Gardiner C, Geldenhuys G, Gott M. Interventions to reduce social isolation and loneliness among older people: an integrative review. Health Soc Care Commun. 2018;26:147-57.

74. Bickerdike L, Booth A, Wilson PM, Farley K, Wright K. Social prescribing: less rhetoric and more reality. A systematic review of the evidence. BMJ Open. 2017;7:e13384.

75. Foster A, Thompson J, Holding E, Ariss S, Mukuria C, Jacques R, et al. Impact of social prescribing to address loneliness: a mixed methods evaluation of a national social prescribing programme. Health Soc Care Commun. 2020. https://doi.org/10.1111/hsc. 13200.

Publisher's Note Springer Nature remains neutral with regard to jurisdictional claims in published maps and institutional affiliations. 